\title{
Avaliação de Sistemas de Recomendação Educacionais no Brasil: uma revisão sistemática da literatura
}

\author{
Janderson J. B. Aguiar ${ }^{1}$, Savyo I. N. Santos ${ }^{1}$, Joseana M. Fechine ${ }^{1}$, Evandro B. Costa ${ }^{2}$ \\ ${ }^{1}$ Universidade Federal de Campina Grande (UFCG), Campina Grande - PB - Brasil \\ ${ }^{2}$ Universidade Federal de Alagoas (UFAL), Maceió - AL - Brasil \\ \{janderson, savyo\}@copin.ufcg.edu.br, joseana@dsc.ufcg.edu.br, evandro@ic.ufal.br
}

\begin{abstract}
In this paper, the authors describe a Systematic Review about Recommender Systems in Education (RSE) focused on the evaluation form used, considering the primary studies published in five Brazilian scientific publication vehicles highest rated by Qualis/CAPES. Besides the evaluation form, the data indicated the historical evolution of these publications, the institutions involved, the metrics most used, and others. With this review, the authors aims that educators and researchers may have easier access to Brazilian initiatives that propose and evaluate in RSE.
\end{abstract}

Resumo. Neste artigo, é descrita uma Revisão Sistemática de Sistemas de Recomendação Educacionais (SRE) com foco na forma de avaliação empregada, considerando os estudos primários divulgados nos cinco veículos brasileiros de publicação científica melhor classificados pelo Qualis/CAPES. Além da forma de avaliação, os dados obtidos indicaram a evolução histórica dessas publicações, as instituições envolvidas, as métricas mais utilizadas, entre outros aspectos. Com esta revisão, espera-se que os educadores e pesquisadores possam ter acesso facilitado às iniciativas que propõem e avaliam SRE no Brasil.

\section{Introdução}

Os Sistemas de Recomendação (SR) surgiram com o objetivo de auxiliar os usuários a acessarem informações relevantes em meio ao crescente número de informações disponíveis [Bobadilla et al. 2013]. No âmbito educacional, é possível ter SR específicos para filtrar recursos contemplando os perfis individuais dos alunos, professores e/ou outros agentes envolvidos na educação, sendo possível a utilização do termo Sistemas de Recomendação Educacionais (SRE) para os SR voltados, de maneira geral, ao processo de ensino e aprendizagem.

Para avaliar SR, em essência, deve-se verificar a satisfação do usuário, pois o interesse está em perceber o grau de aceitação das recomendações — o número de vezes que os usuários aceitam ou rejeitam itens recomendados [Dos Reis 2012]. Adomavicius e Tuzhilin (2005) afirmam que a acurácia dos SR pode ser medida de forma estatística ou por suporte à decisão. Enquanto as métricas estatísticas comparam as avaliações estimadas com as avaliações reais dos usuários, as métricas de suporte à decisão determinam quão bem um SR pode predizer itens que seriam bem classificados pelo usuário [Medeiros 2013].

Dentre as métricas estatísticas mais utilizadas, é possível citar o RMSE (Root Mean Square Error - Raiz do Erro Médio Quadrático) e o MAE (Mean Absolute Error - Erro Médio Absoluto). Dentre as métricas de suporte à decisão, destaca-se o uso da 
Medida F (F-Measure), Precisão (Precision) e Cobertura (Recall). O detalhamento destas e de outras métricas pode ser encontrado no trabalho de Herlocker et al. (2004).

Como defendem Costa, Aguiar e Magalhães (2013), um aspecto que difere bastante os SRE, em relação a outros SR, é a forma de avaliá-los, faltando conjuntos de dados de referência e havendo pouca padronização dos procedimentos de avaliação. Além disso, em se tratando de SRE, há várias dimensões de avaliação possíveis, como a consideração de questões pedagógicas [Manouselis et al. 2012].

No Brasil, o número de iniciativas em SRE tem crescido nos últimos anos [Aguiar et al. 2014]. Além desse crescimento, percebe-se, a partir do que foi exposto anteriormente, que é desafiador e importante avaliar os SRE propostos. Sentiu-se, com isso, a necessidade de organizar uma Revisão Sistemática (RS) da literatura com o objetivo de identificar e analisar o modo como estão sendo avaliados os SRE publicados no Brasil.

O restante do artigo está estruturado da seguinte forma: na Seção 2, são comentados estudos relacionados; na Seção 3, é descrito o método empregado nesta RS; na Seção 4, são mostrados e analisados os resultados; e, na Seção 5, são apresentadas as considerações finais.

\section{Estudos Relacionados}

Em relação a estudos relacionados, Chughtai, Selama e Ghani (2013) publicaram uma RS no âmbito de SRE - considerando estas máquinas de busca: IEEE, ELSEVIER, SCIRUS, IDOSI, AJET, ICVL e SPRINGER - a fim de discutir as abordagens de filtragem da informação utilizadas nos SRE, além dos seus benefícios, limitações e problemas. Este trabalho não dá enfoque na avaliação dos modelos propostos, e, pelas máquinas de busca consideradas, não abrange muitas iniciativas brasileiras.

Com enfoque em veículos brasileiros de publicação científica, há algumas RS realizadas - a exemplo de [Pietruchinski et al. 2011], [Aureliano e Tedesco 2012] e [De Araújo, De Brito e Da Silva 2013], que revisaram, no contexto de eventos em Informática na Educação (IE), pesquisas sobre, respectivamente, jogos educativos, ensino-aprendizagem de programação e softwares para educação inclusiva.

Recentemente, foram realizados dois estudos secundários relativos à recomendação no contexto educacional [Aguiar et al. 2014; Pontes et al. 2014]. Aguiar et al. (2014) mapearam iniciativas nacionais em SRE, indicando sua evolução histórica, as instituições envolvidas, as técnicas de recomendação mais utilizadas, os principais recursos recomendados, entre outros aspectos. Pontes et al. (2014) revisaram as publicações sobre as filtragens de recomendação de objetos de aprendizagem.

Nenhum desses estudos, entretanto, comentou aspectos relacionados às estratégias de avaliação adotadas pelos SRE propostos nesses artigos, o que consiste no foco da RS descrita neste artigo.

\section{Método}

De acordo com Kitchenham (2004), a Revisão Sistemática é um método utilizado para a realização de revisões bibliográficas de forma organizada, com etapas bem definidas, proporcionando maior fundamentação e credibilidade científica. Nesse método, a definição dos objetivos gerais e secundários, além das questões de pesquisa, é 
extremamente importante para o estabelecimento do enfoque de interesse do estudo, uma vez que os objetivos norteiam a RS e as respostas às questões ajudam a obter conclusões e análises específicas em relação ao problema considerado. Além desses pontos, é necessário determinar, no início da pesquisa, critérios de inclusão e exclusão para selecionar os artigos. O conjunto das questões e dos critérios é denominado protocolo de pesquisa [Kitchenham 2004].

Segundo Barros et al. (2008), uma RS tem as seguintes etapas: (1) coleta das publicações potencialmente relevantes sobre a questão analisada, sendo usualmente realizada uma busca em serviços de indexação de publicações em uma determinada área; (2) seleção dentre as publicações coletadas daquelas que satisfazem a um critério de elegibilidade especificado por meio da leitura dos resumos e dos artigos, podendo ser executada em diferentes subetapas, cada vez mais seletivas, e também podendo ser as leituras realizadas por duas ou mais pessoas independentemente, como maneira de se chegar a um consenso na análise; (3) síntese das variações entre os artigos selecionados na etapa anterior, resumindo os resultados desses artigos geralmente em tabelas; (4) opcionalmente, agregação dos resultados individuais de cada publicação em um resultado único, podendo envolver técnicas estatísticas complexas e, por isso, esta fase nem sempre é executada.

A RS descrita neste artigo foi realizada com base no Mapeamento Sistemático (MS) executado por Aguiar et al. (2014). Petticrew e Roberts (2006) afirmam que um MS - também definido como uma pesquisa da literatura - pode identificar quais tipos de estudos podem ser abordados por uma RS. Enquanto o MS possibilita uma visão mais ampla dos estudos primários - com questões de pesquisa mais abrangentes e que podem ser de natureza exploratória e descritiva - a RS possui questões de pesquisas mais definidas, focadas em um ponto específico da área de estudo [Kitchenham e Charters 2007]. Nesse sentido, o MS realizado por Aguiar et al. (2014) despertou a necessidade desta RS com enfoque na avaliação de SRE.

\subsection{Objetivos Gerais e Específicos}

A partir do questionamento central do MS de Aguiar et al. (2014), cujo interesse era saber como os SR estão sendo construídos/utilizados, no Brasil, para apoiar o processo de ensino e aprendizagem, os autores perceberam a necessidade de saber como esses SRE estão sendo avaliados. Considerando o método de RS, foram inicialmente definidos os objetivos gerais e específicos (listados no Quadro 1) para nortear a revisão e servir de base para a construção das questões de pesquisa.

Quadro 1. Objetivos gerais e específicos da RS

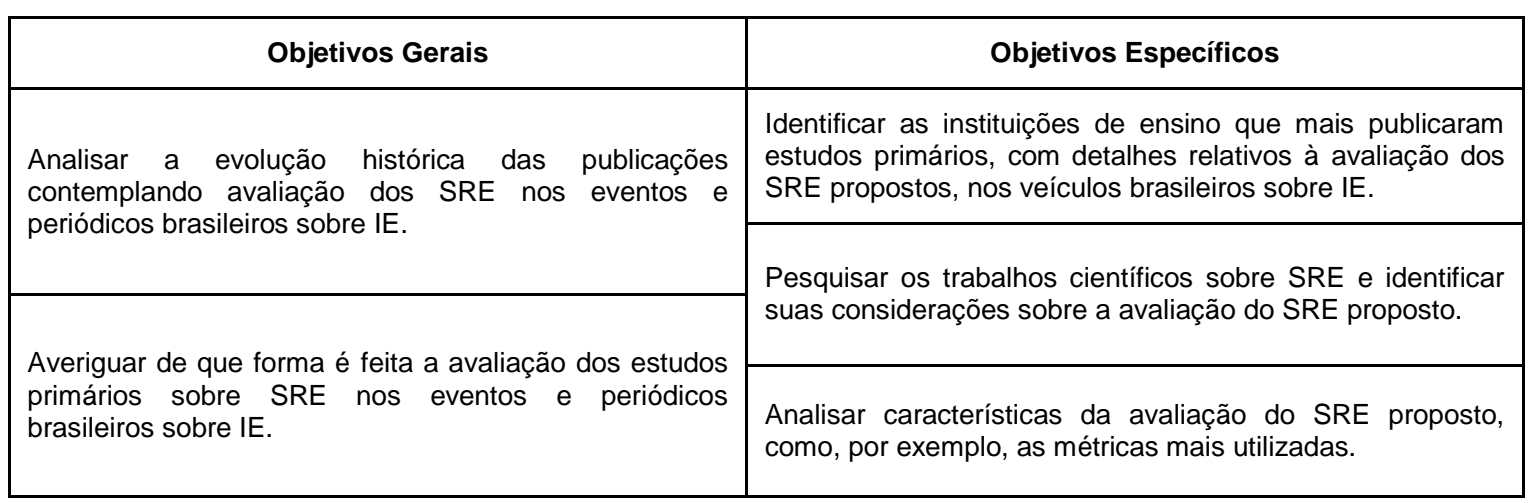




\subsection{Questões de Pesquisa}

Com base nos objetivos apresentados no Quadro 1, foram definidas as seguintes questões de pesquisa para esta RS:

1. Como se deu a evolução histórica das publicações que propõem e avaliam SRE nos eventos e periódicos brasileiros sobre IE?

2. Quais são as instituições que mais publicaram estudos primários que propõem e avaliam SRE nos veículos brasileiros de publicação científica sobre IE?

3. De que forma se deu a avaliação dos SRE propostos nessas pesquisas?

4. Qual(is) a(s) métrica(s) mais utilizada(s) nas avaliações dos SRE no Brasil?

\subsection{Fontes de Busca}

Considerando as questões de pesquisa definidas na subseção 3.2, e seguindo aspectos do protocolo definido por Aguiar et al. (2014), as fontes de busca utilizadas foram os eventos e periódicos do Brasil voltados para a educação e informática/novas tecnologias, com estrato pelo menos B5 na área de avaliação "Ciência da Computação" da classificação Qualis da CAPES (Coordenação de Aperfeiçoamento de Pessoal de Nível Superior). São eles: Informática na Educação: Teoria \& Prática (InfEducTeoriaPratica), Revista Brasileira de Informática na Educação (RBIE), Revista Novas Tecnologias na Educação (RENOTE), Simpósio Brasileiro de Informática na Educação (SBIE) e Workshop de Informática na Escola (WIE).

\subsection{String de Busca}

A string de busca definida no protocolo do MS de Aguiar et al. (2014), e utilizada nesta RS, foi recom*, por ser um radical presente nas 4 línguas que os veículos de busca utilizados contemplam e, além disso, por ser um termo abrangente, evitando perda de possíveis resultados importantes caso se fizesse uma busca mais restrita.

\subsection{Critérios de Seleção}

Os Critérios de Inclusão para a seleção dos artigos desta RS foram:

- A string de busca deve estar presente em qualquer parte dos artigos;

- Os artigos devem ser escritos em inglês, português, espanhol ou francês (idiomas aceitos em pelo menos uma das fontes de busca consideradas);

- A versão completa dos artigos deve estar disponível on-line (considerando esse aspecto, o período de tempo dos artigos publicados variou em relação à fonte de busca - InfEducTeoriaPratica: 1991 a 2014; RBIE: 1997 a 2014; RENOTE: 2003 a 2014; SBIE: 2001 a 2014; WIE: 2003 e 2005 a 2014);

- Os artigos devem tratar sobre recomendação de recursos educacionais, sendo esta funcionalidade o enfoque, ou pelo menos parte em destaque, dos artigos publicados;

- Os artigos devem descrever como a funcionalidade de recomendação do SRE abordado foi avaliada.

Nesse sentido, os Critérios de Exclusão consistiram naturalmente no complemento ou negação dos Critérios de Inclusão. Mas, além disso, foram excluídos artigos que se enquadravam em pelo menos um destes casos:

- Artigos publicados posteriormente em outro veículo considerado na RS; 
- Iniciativas estrangeiras;

- Documentos retornados na busca que não são estudos primários publicados no evento/periódico;

- Short papers (até 5 páginas) ou Full Papers com explicação superficial do processo de avaliação (ou seja, com falta de detalhes para responder às questões de pesquisas desta revisão).

\section{Análise dos Resultados}

Utilizando a string mencionada na subseção 3.4 para buscar estudos primários nas 5 fontes definidas na subseção 3.3, foram retornados inicialmente 361 artigos. Após a triagem inicial (estratégia de exclusão a partir do título, resumo e leitura do artigo usando a técnica scanning), foram selecionados 51 artigos para uma leitura detalhada.

Após a análise minuciosa dos artigos pelos autores, aplicando os critérios de inclusão e exclusão definidos na subseção 3.5, foram selecionados 26 artigos que tratavam de SRE no Brasil, contemplando detalhes de avaliação em sua publicação.

No Quadro 2, são apresentados, por fonte de busca, os resultados por etapa de seleção dos artigos.

Quadro 2. Quantidade de artigos resultantes por etapa e fonte de busca

\begin{tabular}{|c|c|c|c|}
\hline Fontes de busca & $\begin{array}{c}\text { Etapa 1: } \\
\text { Retorno Inicial }\end{array}$ & $\begin{array}{c}\text { Etapa 2: } \\
\text { Triagem Inicial }\end{array}$ & $\begin{array}{c}\text { Etapa 3: } \\
\text { Resultado Final }\end{array}$ \\
\hline InfEducTeoriaPratica & 55 & 3 & 1 \\
\hline RBIE & 14 & 6 & 5 \\
\hline RENOTE & 226 & 13 & 6 \\
\hline SBIE & 59 & 28 & 13 \\
\hline WIE & 7 & 1 & 1 \\
\hline TOTAL & $\mathbf{3 6 1}$ & $\mathbf{5 1}$ & $\mathbf{2 6}$ \\
\hline
\end{tabular}

Os 26 artigos considerados nesta RS encontram-se listados no Quadro 3. As respostas às questões de pesquisa encontram-se nas seguintes subseções.

\subsection{Evolução histórica das publicações}

A evolução histórica das publicações que propõem e avaliam SRE em veículos brasileiros de publicação sobre IE é mostrada na Figura 1.

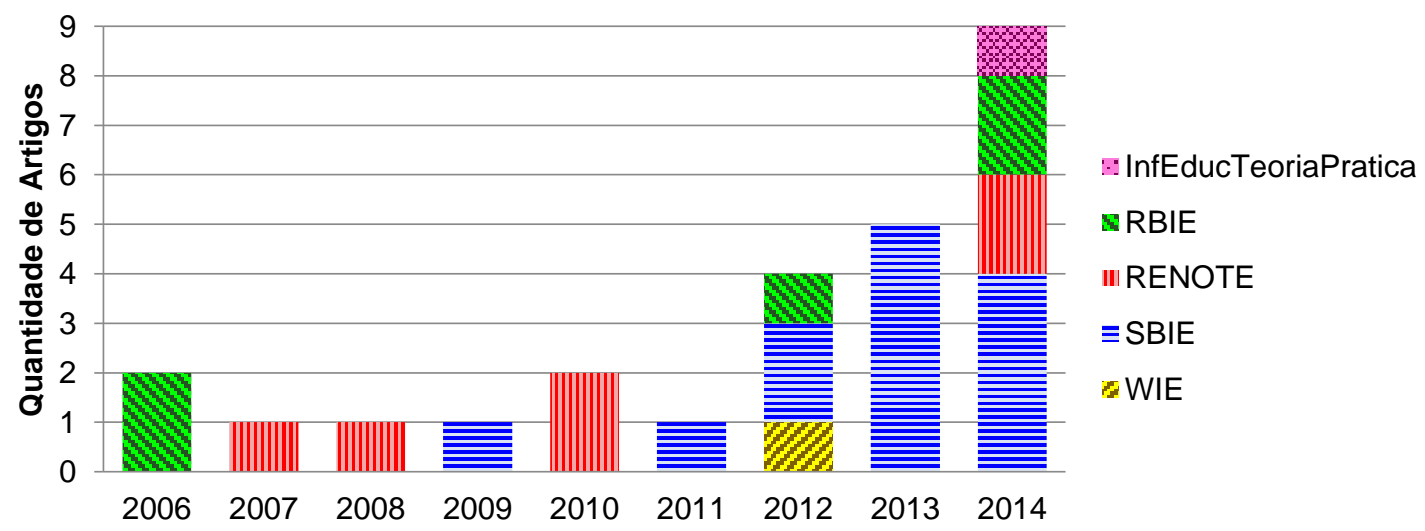

Figura 1. Evolução das Pesquisas por Ano e Fonte de Busca 
CBIE-LACLO 2015

Anais do XXVI Simpósio Brasileiro de Informática na Educação (SBIE 2015)

\section{Quadro 3. Resultado final da RS}

\begin{tabular}{|c|c|}
\hline Título do Artigo & Hiperlink \\
\hline $\begin{array}{l}\text { Conhecendo o usuário através de enriquecimento semântico para } \\
\text { apoio à aprendizagem informal em saúde }\end{array}$ & ie.org/pub/index.php/sbie/article/view/2956/2691 \\
\hline $\begin{array}{l}\text { Desenvolvendo um Sistema de Recomendação de Objetos de } \\
\text { Aprendizagem baseado em Competências para a Educação: relato de } \\
\text { experiências }\end{array}$ & ie.org/pub/index.php/sbie/article/view/1710 \\
\hline $\begin{array}{c}\text { E-BIACS: Um Sistema para a Construção de Ambientes Virtuais para } \\
\text { Aprendizagem Baseada em Problemas }\end{array}$ & $\frac{\underline{\text { http://www.seer.ufrgs.br/index.php/renote/article/view/ }}}{\underline{49832 / 31190}}$ \\
\hline $\begin{array}{c}\text { e-LORS: Uma Abordagem para Recomendação de Objetos de } \\
\text { Aprendizagem }\end{array}$ & ie.org/pub/index.php/rbie/article/view/1289 \\
\hline $\begin{array}{c}\text { LORSys - Um Sistema de Recomendação de Objetos de } \\
\text { Aprendizagem SCORM }\end{array}$ & http://seer.ufrgs.br/index.php/renote/article/view/15223 \\
\hline $\begin{array}{c}\text { O Uso de Técnicas de Recomendação em um Sistema para Apoio à } \\
\text { Aprendizagem Colaborativa }\end{array}$ & http://www.br-ie.org/pub/index.php/rbie/article/view/46 \\
\hline $\begin{array}{l}\text { Podes me ajudar? Apoiando a formação de pares em sistemas de } \\
\text { ajuda em pares através de técnicas de recomendação }\end{array}$ & ie.org/pub/index.php/sbie/article/view/2565 \\
\hline $\begin{array}{l}\text { Predição do Desempenho do Aluno usando Sistemas de } \\
\text { Recomendação e Acoplamento de Classificadores }\end{array}$ & ie.org/pub/index.php/sbie/article/view/2544 \\
\hline $\begin{array}{c}\text { Recomendação assistida por computador de materiais didáticos em } \\
\text { ambientes virtuais de aprendizagem }\end{array}$ & $\frac{\text { http://seer.ufrgs.br/index.php//nfEducTeoriaPratica/arti }}{\underline{\text { cle/view/40301/29979 }}}$ \\
\hline $\begin{array}{c}\text { Recomendação Colaborativa de Conteúdos Educacionais para } \\
\text { Dispositivos Portáteis }\end{array}$ & ie.org/pub/index.php/sbie/article/view/3031/2542 \\
\hline $\begin{array}{c}\text { Recomendação de Jogos na Aprendizagem da Matemática baseado } \\
\text { na Análise Diagnóstica e Teoria de Resposta ao Item }\end{array}$ & ie.org/pub/index.php/sbie/article/view/3032/2543 \\
\hline $\begin{array}{l}\text { Recomendação de Objetos de Aprendizagem Empregando Filtragem } \\
\text { Colaborativa e Competências }\end{array}$ & $\begin{array}{c}\text { http://www.br- } \\
\text { ie.org/pub/index.php/sbie/article/view/1158 }\end{array}$ \\
\hline $\begin{array}{l}\text { Recomendando Objetos de Aprendizagem a partir das hashtags } \\
\text { postadas no Moodle }\end{array}$ & ie.org/pub/index.php/sbie/article/view/2486 \\
\hline $\begin{array}{c}\text { Relato de uma Experiência de Auxílio Personalizado a Alunos em } \\
\text { Ambiente Virtual de Aprendizagem Utilizando Agentes }\end{array}$ & ie.org/pub/index.php/wie/article/view/2122 \\
\hline Sistema de Recomendação Acadêmico para Apoio a Aprendizagem & http://seer.ufrgs.br/index.php/renote/article/view/14236 \\
\hline $\begin{array}{l}\text { Sistema de Recomendação de Links para o fomento de discussões em } \\
\text { fóruns de um Ambiente Virtual de Aprendizagem }\end{array}$ & $\frac{\text { http://www.seer.ufrgs.br/index.php/renote/article/view/ }}{\underline{53547 / 33050}}$ \\
\hline $\begin{array}{c}\text { Sistema de Recomendação Híbrido para Bibliotecas Digitais que } \\
\text { Suportam o Protocolo OAI-PMH }\end{array}$ & ie.org/pub/index.php/sbie/article/view/1584 \\
\hline $\begin{array}{c}\text { Técnica de Recomendação Baseada em Metadados para Repositórios } \\
\text { Digitais Voltados ao Ensino }\end{array}$ & ie.org/pub/index.php/sbie/article/view/2546 \\
\hline Um Agente Animado para Ambientes de Aprendizagem Colaborativos & http://www.br-ie.org/pub/index.php/rbie/article/view/27 \\
\hline $\begin{array}{l}\text { Um Modelo de Recomendação Ubíqua de Conteúdo para Grupos de } \\
\text { Aprendizes }\end{array}$ & ie.org/pub/index.php/sbie/article/view/2548 \\
\hline $\begin{array}{l}\text { Um Modelo Multiagente para Recomendação de Conteúdo } \\
\text { Educacional em um Ambiente Ubíquo }\end{array}$ & ie.org/pub/index.php/sbie/article/view/1782 \\
\hline $\begin{array}{l}\text { Um Modelo para Recomendação de Artigos Acadêmicos Baseado em } \\
\text { Filtragem Colaborativa Aplicado à Ambientes Móveis }\end{array}$ & http://seer.ufrgs.br/index.php/renote/article/view/14458 \\
\hline $\begin{array}{l}\text { Um Modelo para Recomendação de Objetos de Aprendizagem } \\
\text { Utilizando Similaridade de Sessões }\end{array}$ & ie.org/pub/index.php/rbie/article/view/2849/2834 \\
\hline $\begin{array}{l}\text { Um Serviço para Recomendação de Artigos Científicos Baseado em } \\
\text { Filtragem de Conteúdo Aplicado a Dispositivos Móveis }\end{array}$ & http://seer.ufrgs.br/index.php/renote/article/view/18057 \\
\hline $\begin{array}{l}\text { Uma Abordagem Automática para Personalização do Processo de } \\
\text { Ensino Baseada em Estilos de Aprendizagem em Sistemas } \\
\text { Adaptativos e Inteligentes para Educação a Distância }\end{array}$ & ie.org/pub/index.php/rbie/article/view/2374/2724 \\
\hline $\begin{array}{l}\text { Uma Abordagem para Recomendação Automática e Dinâmica de } \\
\text { Objetos de Aprendizagem Baseada em Estilos de Aprendizagem }\end{array}$ & ie.org/pub/index.php/sbie/article/view/3065/2573 \\
\hline
\end{tabular}


É visto, a partir da Figura 1, que a maior parte das publicações, sobretudo nos três últimos anos, ocorreram no SBIE, considerado o evento mais importante na área de IE no Brasil. Além disso, percebe-se, com o passar dos anos, um crescimento no número de publicações sobre SRE, em eventos e periódicos brasileiros sobre IE, e que destacam a fase de avaliação do SRE proposto.

\subsection{Instituições que mais publicaram artigos}

As instituições que publicaram trabalhos científicos com propostas e avaliações de SRE, nos veículos brasileiros de publicação sobre IE consideradas nesta revisão, são apresentadas na Figura 2.

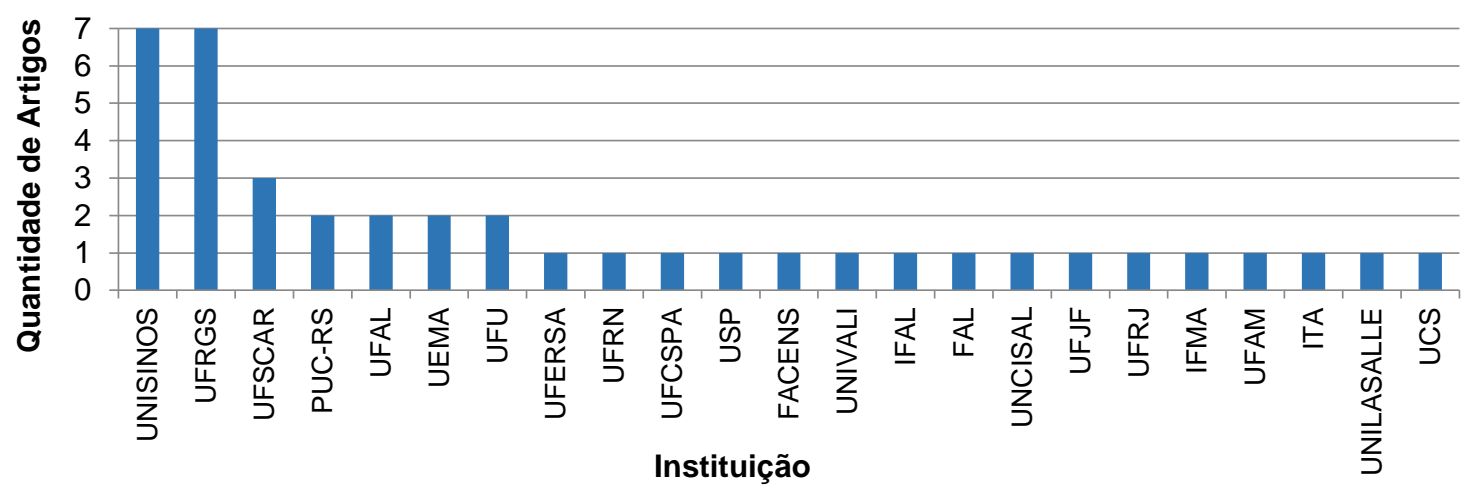

Figura 2. Quantidade de Publicações por Instituição

Como indicado no gráfico, as instituições que se destacaram foram a UNISINOS e a UFRGS. É importante mencionar que 38\% das publicações adviram de parcerias entre instituições distintas e geralmente do mesmo estado - IFAL, UFAL, UNCISAL e FAL; IFMA e UEMA; UFERSA e UFRN; UFRGS e PUC-RS; UFRGS e UCS; UNISINOS e UFRGS; UNISINOS e UFRGS; UNISINOS, UFCSPA e UFRGS; USP, UFSCAR e FACENS. Além dessas, houve uma parceria entre UNILASALLE, UFRGS (ambas do Rio Grande do Sul) e ITA (estado de São Paulo).

Observou-se, portanto, que a região do país que abrange mais publicações nessa linha de pesquisa, preocupando-se com a avaliação dos SRE, é a região Sul, seguida das regiões Sudeste, Nordeste e Norte.

\subsection{Tipo de avaliação realizada}

Em relação ao modo como foi realizada a avaliação dos SRE abordados nos estudos selecionados nesta RS, percebeu-se que é feito uso de estratégias empíricas e, portanto, os artigos foram categorizados em: (i) Experimento - controlados ou observacionais (estudos de caso); e/ou (ii) Survey — utilização de entrevistas/questionários dependendo da memória e opinião dos participantes. A distribuição dos artigos nessas categorias é apresentada na Figura 3.

Além de experimentos controlados, foi percebida a realização de muitos estudos de caso (também denominados experimentos observacionais) e, especialmente em relação a estes, o uso frequente de questionários para avaliar o SRE proposto. Apesar de considerarem essa estratégia de avaliação, há artigos que não descrevem bem o conteúdo desses questionários, dificultando a clareza dos aspectos avaliados $\mathrm{e}$ identificação de métricas empregadas, o que impactou negativamente na objetividade da resposta para a quarta questão de pesquisa (subseção 3.2). 


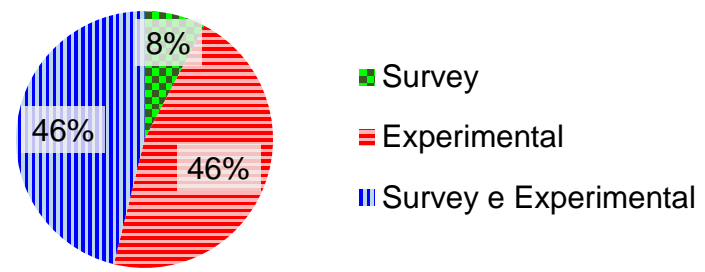

Figura 3. Distribuição das pesquisas em relação ao modo de avaliação

\subsection{Métricas mais utilizadas nas avaliações}

Em se tratando das métricas utilizadas nas avaliações das iniciativas brasileiras em SRE no Brasil, com base no exposto na seção 1, os artigos foram categorizados inicialmente pelo tipo de métrica: (i) Estatística; (ii) De suporte à decisão, e (iii) Outro — esta categoria foi pensada como estratégia para especialmente os casos de artigos que utilizaram survey como estratégia empírica, devido à dificuldade mencionada na subseção 4.3. Na figura 4a é mostrada a distribuição dos artigos nessas categorias.

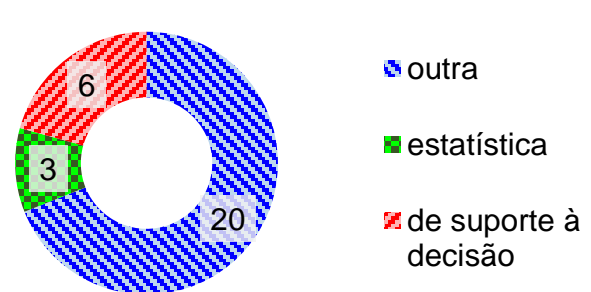

(a)

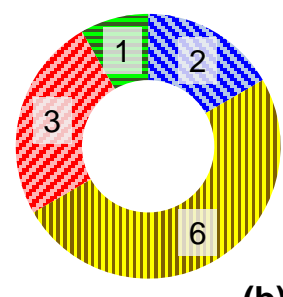

(b) $\triangle M A E$

II Precision

o Recall

EF-Measure

Figura 4. Distribuição das pesquisas com relação a: (a) tipo de métricas; (b) métricas populares estatísticas e/ou de suporte à decisão

Apesar do gráfico da Figura 4a totalizar 29 artigos, em vez de 26, é importante comentar que dois artigos utilizaram concomitantemente métricas estatísticas e de suporte à decisão, além de outro artigo utilizar métricas de suporte à decisão e outra.

A partir disso, foi gerado um gráfico (Figura $4 b$ ) para analisar o uso das métricas estatísticas e/ou de suporte à decisão mais amplamente utilizadas na avaliação de SR (conforme comentado na seção 1). Verificou-se portanto que, dentre elas, a métrica Precisão (Precision) foi a mais utilizada.

Analisando a Figura 4, constata-se, como defendido por alguns autores [Costa, Aguiar e Magalhães 2013] [Manouselis et al. 2012], a falta de padronização no modo de avaliar SRE, especialmente devido a aspectos importantes no âmbito educacional, como a avaliação da interface, destacada em alguns trabalhos.

Devido à limitação do tamanho deste artigo, não foi possível explanar uma análise mais meticulosa dos resultados, como, por exemplo, uma discussão relativa à qualidade dos artigos retornados. Em contrapartida, neste hiperlink http://goo.gl/IlNp4u encontram-se detalhes relativos a cada um dos 26 trabalhos - o usuário-alvo da recomendação, o recurso educacional recomendado, as técnicas de recomendação empregadas, as métricas de avaliação, entre outras informações.

\section{Considerações Finais}

A RS descrita neste artigo permitiu elencar e analisar as iniciativas em SRE, sob o aspecto da avaliação empregada, considerando as publicações dos eventos e periódicos brasileiros mais importantes na área de IE e Novas Tecnologias. 
Com esta revisão, foi constatado que os estudos utilizaram, em sua maioria, uma estratégia empírica experimental, mas que combinaram muitas vezes com a estratégia de survey. Os autores acreditam que, mesmo havendo aspectos mais subjetivos e específicos no âmbito educacional, geralmente captados via opinião dos usuários em surveys, é importante que as informações via questionários (ou meios afins) sejam sempre disponibilizadas com clareza nas publicações. Além disso, os autores deste artigo defendem que, por se tratar de um SR, é sempre interessante empregar também métricas conhecidas de suporte à decisão e/ou estatísticas.

Drachsler et al. (2015) defendem que as pesquisas sobre SRE deveriam seguir um método de avaliação padronizado, como estes quatro passos sugeridos por Manouselis et al. (2012): (i) uma seleção de conjuntos de dados que se adequam ao problema de recomendação e às tarefas do desenvolvimento; (ii) um estudo de comparação off-line de diferentes algoritmos em relação aos conjuntos de dados selecionados, incluindo conjuntos de dados conhecidos para fornecer insights sobre o desempenho dos algoritmos de recomendação; (iii) um estudo abrangente do usuário em um ambiente experimental controlado para testar os efeitos psico-educativos em relação aos alunos, bem como testar os aspectos técnicos do SR projetado; e (iv) a implantação do SR em uma aplicação real, na qual possa ser testado em condições operacionais realistas/normais com seus usuários legítimos. Além disso, Drachsler et al. (2015) comentam que é importante que esses quatro passos sejam publicados junto com uma descrição completa do SR, sendo declarado e acessível ao público o conjunto de dados utilizado, permitindo que outros pesquisadores repitam e ajustem qualquer parte da pesquisa para obter resultados comparáveis e novos insights.

De maneira geral, conclui-se que os estudos primários retornados nesta RS podem servir como referência para a criação de novos SRE, possibilitando aos pesquisadores e educadores - por meio dos resultados e do acesso facilitado aos artigos - conhecer as iniciativas que propõem e avaliam sistemas com estratégias de recomendação de recursos educacionais no Brasil.

Ressalta-se que, por considerar apenas as fontes de busca definidas na subseção 3.3, esta RS possui uma limitação em relação à abrangência de trabalhos primários considerados, uma vez que podem haver iniciativas brasileiras importantes que avaliaram SRE em outras fontes de busca. Todavia, decidiu-se manter aspectos do protocolo de pesquisa empregado anteriormente [Aguiar et al. 2014], focando na questão de avaliação, dado que assim seria (e foi) possível vislumbrar as avaliações em SRE de trabalhos publicados nos eventos e periódicos brasileiros mais importantes em IE.

Em relação a esse aspecto, vale destacar que, para as edições de 2015 do SBIE e do WIE, foi adotada pela Organização Geral e pelo Comitê Científico a estratégia de publicar artigos completos de apenas estudos primários que apresentem explicitamente metodologia e análise de resultados. Com isso, acredita-se que os pesquisadores que pretendem publicar seus modelos de SRE nesses eventos, bastante importantes na área de IE, irão se preocupar mais fortemente em realizar e explanar nos artigos uma avaliação detalhada de suas pesquisas.

Sobre trabalhos futuros, os autores estão utilizando os resultados desta RS como parte do embasamento teórico para planejar a avaliação de alguns modelos de SRE a serem posteriormente publicados. 


\section{Referências Bibliográficas}

Adomavicius, G. e Tuzhilin, A. (2005). Toward the next generation of recommender systems: A survey of the state-of-the-art and possible extensions. Knowledge and Data Engineering, IEEE Transactions on, v. 17, n. 6, p. 734-749.

Aguiar, J. J. B., Santos, S. I. N., Fechine, J. M. e Costa, E. B. (2014). Um Mapeamento Sistemático sobre Iniciativas Brasileiras em Sistemas de Recomendação Educacionais. In: XXV Simpósio Brasileiro de Informática na Educação (SBIE), p. 1123-1132.

Aureliano, V. C. O. e Tedesco, P. C. A. R. (2012). Ensino-aprendizagem de Programação para Iniciantes: uma Revisão Sistemática da Literatura focada no SBIE e WIE. In: Anais do $23^{\circ}$ Simpósio Brasileiro de Informática na Educação (SBIE), Rio de Janeiro.

Barros, A. C., Wainer, J., Claudio, K., Ferreira, L. R. R. e Dwyer, T. (2008). Uso de Computadores no Ensino Fundamental e Médio e Seus Resultados Empíricos: Uma Revisão Sistemática da Literatura. In: Revista Brasileira de Informática na Educação (RBIE), v. 16, n. 1, p. 57-68.

Bobadilla, J., Ortega, F., Hernando, A. e Gutiérrez, A. (2013). Recommender systems survey. In: Knowledge Based Systems, v. 46, p. 109-132.

Chughtai, W. M., Selama, A. B. e Ghani, I. (2013) Short Systematic Review on E-Leaning Recommender Systems. In: Journal of Theoretical and Applied Information Technology, v. 57, n. 2.

Costa, E., Aguiar, J. e Magalhães, J. (2013). Sistemas de Recomendação de Recursos Educacionais: conceitos, técnicas e aplicações. In: II Congresso Brasileiro de Informática na Educação - Jornada de Atualização em Informática na Educação (JAIE), p. 57-78.

De Araújo, A. L. S. O., De Brito, R. R. e Da Silva, A. P. (2013). Softwares para Educação Inclusiva: Uma Revisão Sistemática no Contexto de SBIE e WIE. Anais do $24^{\circ}$ Simpósio Brasileiro de Informática na Educação (SBIE), Campinas, São Paulo.

Dos Reis, L. F. M. (2012). Sistema de Recomendação Baseado em Conhecimento. 110 f. Dissertação (Mestrado em Engenharia Informática) - Faculdade de Ciências e Tecnologia, Universidade de Coimbra, Coimbra.

Drachsler, H., Verbert, K., Santos, O. C. e Manouselis, N. (2015). Panorama of recommender systems to support learning. Handbook on recommender systems. (no prelo). Disponível em: $<$ https://lirias.kuleuven.be/bitstream/123456789/476545/1/TEL_RecSys.pdf $>$. Acesso em 29/05/2015.

Herlocker, J. L., Konstan, J. A., Riedl, J. T. e Terveen, L. G. (2004). Evaluating collaborative filtering recommender systems. ACM Transactions on Information Systems 22(1), p. 5-53.

Kitchenham, B. (2004). Procedures for performing systematic reviews. Relatório Técnico, Keele University and NICTA.

Kitchenham, B. e Charters, S. (2007). Guidelines for performing Systematic Literature Reviews in Software Engineering. Keele University and Durham University Joint Report.

Manouselis, N., Drachsler, H., Verbert, K. e Duval, E. (2012). Recommender Systems for Learning. Berlin, Springer, $90 \mathrm{p}$.

Medeiros, I. R. G. (2013). Estudo sobre Sistemas de Recomendação Colaborativos. 30 f. Trabalho de Conclusão de Curso (Graduação em Ciência da Computação) - Centro de Informática, Universidade Federal de Pernambuco, Recife.

Petticrew, M. e Roberts, H. (2006) Systematic reviews in the social sciences: A practical guide. Malden, MA: Blackwell Publishing.

Pietruchinski, M. H., Neto, J.C., Malucelli, A. e Reinehr, S. (2011). Os jogos educativos no contexto do SBIE: uma revisão sistemática de Literatura. In: XXII Simpósio Brasileiro de Informática na Educação (SBIE), p. 476-485.

Pontes, W. L., França, R. M., Costa, A. P. M. e Behar, P. (2014). Filtragens de Recomendação de Objetos de Aprendizagem: uma revisão sistemática do CBIE. In: XXV Simpósio Brasileiro de Informática na Educação (SBIE), p. 549-558. 